\title{
Real-time guidance for cardiopulmonary resuscitation in Mixed Reality
}

\author{
Danilo Gasques Rodrigues \\ Dept. of Computer Science and \\ Engineering \\ University of California, San Diego \\ La Jolla, CA, 92037, USA \\ gasques@ucsd.edu
}

\author{
Janet G. Johnson \\ Dept. of Computer Science and \\ Engineering \\ University of California, San Diego \\ La Jolla, CA, 92037, USA \\ jgj007@ucsd.edu
}

\author{
Nadir Weibel \\ Dept. of Computer Science and \\ Engineering \\ University of California, San Diego \\ La Jolla, CA, 92037, USA \\ weibel@ucsd.edu
}
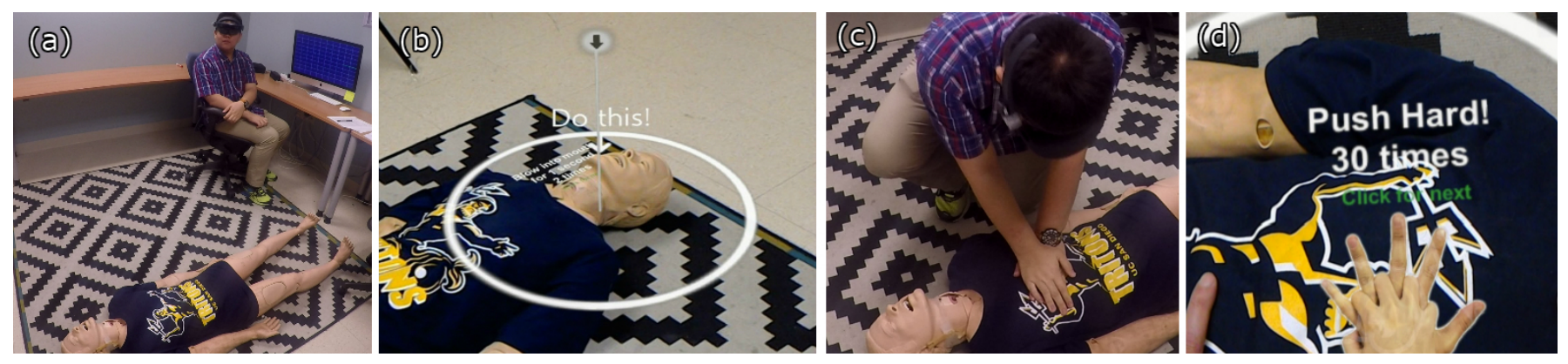

Figure 1: HoloCPR in Action. (a-b) As soon as the simulation manikin heart's stops beating, the user looks at it to find spatially localized information of what he needs to do anchored where it he needs to perform it. (c-d) Wearer is performing chest compressions while seeing the instructions in place.

\begin{abstract}
Cardiopulmonary Resuscitation (CPR) is a life-saving procedure that gives a cardiac arrest victim a second chance. The application window is short as the chances of survival falls drastically with every minute without resuscitation. Outofhospital cardiac arrests are even riskier, as the state of victim worsens while waiting for specialized medical personnel. CPR is simple and composed of few steps, with instructions widely available in First Aid Kits or online. Nonetheless, it is usually not performed by most bystanders as they fear to harm the victim by its incorrect application. Recently, the introduction of Mixed Reality (MR) devices such as Microsoft HoloLens enabled the development of systems that leverage contextual information such as the location of the victim to display guiding information where it should be performed, thereby allowing bystanders to focus on the task at hand. In this demo, we present HoloCPR, a Mixed Reality application that provides real-time instructions for performing CPR.
\end{abstract}

\section{ACM Classification Keywords}

H.5.m Information interfaces and presentation (e.g., HCI)

\section{Author Keywords}

Mixed Reality, Augmented Reality, HoloLens, CPR, Checklists, User-Centered Design

\section{INTRODUCTION AND MOTIVATION}

In the event of an out-of-hospital cardiac arrest, performing cardiopulmonary resuscitation (CPR) immediately after the victim collapses is critical for his or her survival. It often can take 8 minutes or longer for medical personnel to arrive on the scene. Since the chances of survival fall by $7-10 \%$ for every minute without resuscitation, bystander-initiated CPR has the potential to save lives.

Bystanders are people that happen to be around the victim in the event of a sudden cardiac arrest. They are most likely not trained medical professionals and have to rely on past training or their knowledge to help the victim. Although it has been shown that bystander-initiated CPR can double or triple survival rates [3], only 30-40\% [4] of bystanders administer CPR. Factors such as fear of harming the victim or panic influences their readiness to act despite prior training[1].

In an effort to encourage bystander-CPR, first-aid kits often provide manuals that aid performing CPR by providing stepby-step instructions. Alternatively, bystanders can use a mobile phone to provide guidance. Despite making CPR instruc- 
tions more readily available, both alternatives still rely on bystanders' ability to read and interpret the instructions before helping the victim. This potentially increases their cognitive load in an already stressful situation.

With the use of Mixed Reality headsets, such as Microsoft HoloLens ${ }^{1}$, guidance for emergency procedures can become embedded in the real world. By leveraging context to present deictic instructions in real time to the user when and where they need them (Figure 1), we expect bystanders to be able to respond faster to a sudden cardiac arrest.

This demonstration aims at showing HoloCPR, a Mixed Reality interface targeted at helping bystanders that are not trained in Cardiopulmonary Resuscitation (CPR) to intervene and follow a correct procedure to resuscitate a person. Albeit still a prototype, we foresee that it could improve bystander-CPR rates in the near future.

\section{HOLOCPR}

HoloCPR is a Mixed Reality application developed in Unity $3 \mathrm{D}^{2}$ for Microsoft HoloLens. It has two main parts. The first one revolves around the physical resuscitation, and the second one aids the placement of an Automatic Electronic Defibrillator.

HoloCPR uses physical elements associated with the task at hand to display the instructions that should be performed where they should be performed (Figure 1, d). Thus, the environment gives context to instructions, allowing them to be short and deictic. As an example, Figure 1 (d) shows how HoloCPR communicates chest compressions with only four words and a picture. Figure 2 shows the alternative to Mixed Reality interface: a paper checklist. Notice how the text is lengthened by the need to communicate the location of the procedure. Moreover, notice how the instructional picture shows the task from a third person perspective whereas HoloCPR (Figure $1(d)$ ) shows it from a first-person perspective.

Similar to how mapping services (e.g., Google Maps) turn drivers that are new to a city into navigation experts, we expect HoloCPR to increase bystanders' confidence in their ability to perform CPR during emergency procedures. In case of a cardiac arrest, they only have to wear HoloLens and launch HoloCPR. By looking at the victim, the system automatically indicates where to act first (see Fig 1, b), enabling users to address the task at hand. Similar to navigation instructions, steps are revealed as users progress in the rescue procedure.

The system was designed around the Red Cross CPR checklist ${ }^{3}$ by breaking down instructions into "What", "How", and "Where" users should perform them (Figure 2). The "Where" is a crucial piece of information as it defines the real-world location HoloCPR uses to fix the instructions (Figure 1).

\section{DEMONSTRATION}

While a separate paper [2] describes our iterative design process and evaluates the system, this demonstration will let attendees try the system with a medical manikin. They will

\footnotetext{
${ }^{1}$ https://www.microsoft.com/en-us/hololens

${ }^{2}$ https://unity3d.com

${ }^{3}$ https://www.redcross.org
}

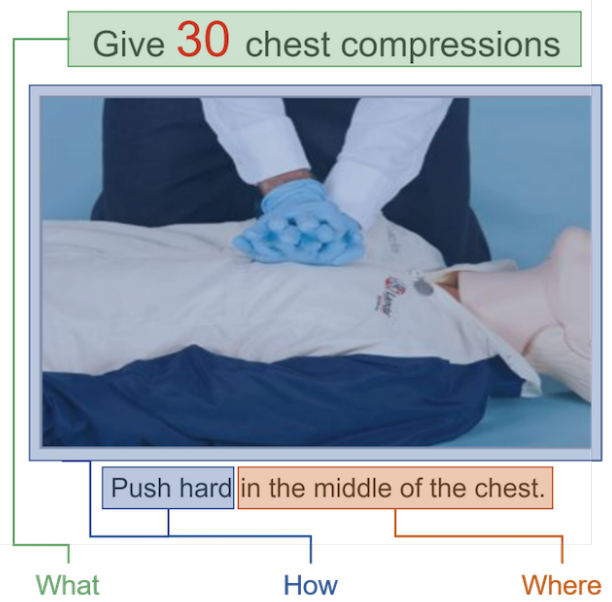

Figure 2: Dividing instructions into "What", "How", and "Where"

follow guidance from HoloCPR to perform steps like chest compressions and rescue breaths. When available or needed, HoloCPR will guide them to the nearest Automatic Electronic Defibrillator so that they can resuscitate the manikin. We will use props to the highest degree of realism possible to increase authenticity while not causing any harm to attendees. We will also let attendees know that HoloCPR is not an approved alternative to CPR training and that trying the application does not entitle them to reproduce the same steps in a real-life emergency.

\section{CONCLUSION}

In this demo we present HoloCPR, an application targeted at supporting novices in performing cardiopulmonary resuscitation (CPR) through an intuitive Mixed Reality interface. Our system guides wearers through different steps, outlining "what" needs to be done, "where" and "how"(Figure 2). The accompanying paper [2] describes how we designed and evaluated HoloCPR.

\section{REFERENCES}

1. R. Graham, M. A. McCoy, A. M. Schultz, et al. The public experience with cardiac arrest. National Academies Press (US), 2015.

2. J. Johnson, D. Gasques Rodrigues, G. Madhuri, and N. Weibel. Holocpr: Designing and evaluating a mixed reality interface for time-critical emergencies. In pervasive computing technologies for healthcare (PervasiveHealth), 2018 12th international conference on, page To appear. ACM, 2018.

3. A. Nielsen, D. Isbye, F. Lippert, and L. Rasmussen. Can mass education and a television campaign change the attitudes towards cardiopulmonary resuscitation in a rural community? SJTREM, 21(1):39, 2013.

4. J. Nolan, J. Soar, and H. Eikeland. The chain of survival. Resuscitation, 71(3):270-271, 2006. 